\title{
Heidegger y el despertar: Una articulación de su perspectiva del obrar y de la praxis en 1929-1930
}

\author{
Heidegger and the Awakening: \\ An Articulation of His Perspective of Act and \\ Praxis 1929-1930 \\ LUIS FERNANDO BUTIERREZ \\ UNIVERSIDAD NACIONAL DE LA PLATA
}

Recibido: 26/01/2020 Aceptado:18/05/2020

\begin{abstract}
RESUMEN
En este trabajo indagaremos en torno al estatuto de la acción y la praxis en la obra de Heidegger a partir de un análisis de la figura del despertar relativa al aburrimiento profundo, en su trabajo de 1929-1930. Con ello, buscaremos demostrar que las implicancias relativas al actuar, pensar y filosofar allí expuestas, permiten cotejar continuidades y diferencias de tipo fronterizo tanto con sus elaboraciones anteriores, como respecto a su perspectiva posterior a la Kehre. Para dar cuenta de ello, primero abordaremos aspectos fundamentales de su interpretación de la filosofía práctica aristotélica y sus reelaboraciones hasta Sein und Zeit.
\end{abstract}

PALABRAS CLAVE

HEIDEGGER, PRAXIS, DESPERTAR, ABURRIMIENTO, MUNDO

\begin{abstract}
In this work we will inquire about the statute of action and praxis in Heidegger's work from an analysis of the figure of awakening relative to deep boredom, in his 1929-1930 work. With this, we will seek to demonstrate that the implications related to acting, thinking and philosophizing exposed there, allow for cross-border continuities and differences to be compared both with their previous elaborations, and with respect to their post-Kehre perspective. To account for
\end{abstract}

(C) Contrastes. Revista Internacional de Filosofía, vol. XXV N² (2020), pp. 99-117. ISSN: 1136-4076

Departamento de Filosofía, Universidad de Málaga, Facultad de Filosofía y Letras Campus de Teatinos, E-29071 Málaga (España) 
this, we will first address fundamental aspects of his interpretation of Aristotelian practical philosophy and his reworkings to Sein und Zeit.

KEYWORDS

HEIDEGGER, PRAXIS, AWAKENING, BOREDOM, WORLD

EN LOS ÚLTIMOS AÑOS, los análisis e interpretaciones sobre la perspectiva de la acción o la teoría de la praxis en las elaboraciones de Heidegger se han desarrollado desde un diálogo con los trabajos de Volpi, en torno a la recepción heideggeriana de textos fundamentales de Aristóteles. En esa línea, las indagaciones respectivas en el período posterior a la Kehre se han desplegado en el contexto de sus análisis en torno al mundo de la técnica y el carácter prospectivo del pensar. En el arco de estas tematizaciones, podemos distinguir la constelación temática Acción-Mundo-Dasein, en especial, desde dos enfoques: uno orientado a la analítica en la década de 1920 y otro, al marco relacional Dasein-Ser en sus trabajos desde 1930.

En este marco, la pregunta por la medida en que una teoría de la praxis heideggeriana encuentra una articulación específica en sus elaboraciones de madurez orienta gran parte de tales análisis de su obra. ${ }^{1}$ Frente a ello, las distinciones entre la dimensión óntica y ontológica de la praxis se articula en diversos debates contemporáneos en torno a la ausencia de una perspectiva práctica y ética concreta en este período, lo cual suele ser entendido en continuidad con la adhesión de Heidegger al Nacional Socialismo, su silencio respectivo y su retirada de los asuntos públicos en los años 30 y $40 .^{2}$ En este caso, tales interpretaciones cuentan entre sus antecedentes con las ya clásicas investigaciones y lecturas de Víctor Farías.

En el siguiente ensayo proponemos una indagación entre tales períodos de las elaboraciones de Heidegger, con un carácter de tipo fronterizo. Específicamente, proponemos articular la pregunta por la perspectiva de la acción y de la praxis en su obra, a partir del análisis del aburrimiento profundo en su seminario de 1929-1930, poniendo el foco en el tratamiento que allí desarrolla en torno a la figura metafórica del despertar.

A nuestro entender, en este análisis se evidencia un pasaje en transición que va de un enfoque centrado en la analítica del Dasein, al de la relación Dasein-ser en el horizonte del Ereignis. Nuestra hipótesis e interpretación

1 Por ejemplo, Thurnher 1991, pp.136s.; Escudero 2010, pp.284-90.

2 Cf. F. de Lara 2014, pp.78s.; Calle Zapata 2016, pp.60ss; Di Pego 2019, pp.213-19, entre otros. 
consiste en sostener que las implicancias relativas al actuar, el pensar y filosofar allí expuestas, permiten cotejar continuidades y diferencias con sus elaboraciones anteriores, ofreciendo elementos para precisar el estatuto de la praxis y sus desplazamientos en su perspectiva posterior.

Para comenzar a fundamentar esta lectura, en primer lugar, abordaremos aspectos fundamentales de su interpretación y reelaboración de la praxis aristotélica, sin descuidar su propia modalidad de abordaje filosófico. Desde allí, analizaremos con mayor detenimiento sus consideraciones prácticas en torno a la necesidad epocal de despertar un temple anímico fundamental para desplegar las preguntas metafísicas fundamentales, en su seminario de 19291930. En ambos casos, por medio de un conjunto de notas, buscaremos dar cuenta de discusiones y contrastes dentro de la obra de Heidegger. Finalmente, una vez especificadas las funciones que atribuye a la labor filosófica en su relación irreductible con la situación histórica, con el mundo y el sí- mismo, buscaremos cotejar en qué medida puede reconocerse allí una continuidad con sus desarrollos anteriores y vías de transición respecto de su enfoque posterior. Por esta vía, nos proponemos contribuir con los debates actuales en torno al estatuto de la acción y concepto de la praxis en la obra de Heidegger.

\section{CONSIDERACIONES SOBRE LA PRAXIS Y EL PROCEDER FILOSÓFICO ENTRE}

$$
\text { 1921-1927 }
$$

Si abordamos los primeros trabajos de Heidegger en los años 20 podemos reconocer su interés creciente por la orientación de la práctica filosófica hacia la vida y la facticidad. ${ }^{3}$ En efecto, desde el conjunto de lecciones impartidas en Friburgo, Heidegger se orienta a la búsqueda de una filosofía autentica, no afectada por los prejuicios de la tradición, que inicialmente denomina como ciencia originaria de la vida (Urwissenschaft) y luego circunscribe en una incipiente hermenéutica de la facticidad. En tal enfoque, la filosofía debe ocuparse de la existencia inmediata en el mundo de la vida fáctica, lo que luego se articula en el desarrollo de las estructuras del Dasein hacia Sein und Zeit (SuZ). En este trayecto del pensar, Heidegger distingue que el vivenciar del mundo se manifiesta entramado en significaciones.

En este contexto, en su manuscrito denominado Natorp Bericht (1922), subraya la importancia de un abordaje de los conceptos tradicionales

3 En este apartado, consideramos los siguientes trabajos: Phänomenologische Interpretation ausgewählter Abhandlungen des Aristoteles zu Ontologie und Logik (Anzeige der hermeneutischen Situation) (1922); Ontologie. Hermeneutik der Factizität (1923); Grundbegriffe der aristotelischen Philosophie (1924); Platon: Sophistes (1924-1925) y Sein und Zeit (1927). En este trabajo, las citas de la obra de Heidegger se consignan primero desde su paginación original en GA y luego en su respectiva traducción. 
para deshacer el estado de interpretación heredado, distinguiendo sus encubrimientos y desvíos respecto de las experiencias y funciones expresivas originarias. ${ }^{4}$ Estas modalidades de abordaje de la tradición, denominadas destrucción (Destruktion) (Heidegger 1921-1922, pp.368 [50s.]) y desmontaje (Abbau) (Heidegger 1923, p.75ss. [99ss]), remiten a un tratamiento práctico de la herencia tradicional, diferenciando aquello que aún es relevante para nuestra situación y apropiado para descubrir la estructura originaria de la vida fáctica.

Aquellas tramas significativas de la vida y esta modalidad de abordaje filosófico se ponen de manifiesto con sus interpretaciones destructivas de la filosofía práctica aristotélica, especialmente en su consideración de la Ética a Nicómaco. ${ }^{5}$ Desde estas interpretaciones desarrolla un enfoque de los tipos de acción, lo cual confluye en lo que Volpi denomina como una ontologización de la praxis (Volpi 1996, pp.46ss).

En este apartado abordaremos cuestiones fundamentales en estos tramos del pensar heideggeriano teniendo en cuenta la relevancia que han tenido sus interpretaciones de los textos de Aristóteles entre los años 1922-1925, tanto en la recepción de su obra, como en los análisis relativos al obrar y la praxis. ${ }^{6}$ En este marco, pondremos atención a sus reelaboraciones y torsión ontológica respectiva, en vistas de su relevancia para la indagación filosófica. En continuidad con los análisis de Volpi, buscaremos distinguir elementos para las relaciones entre Acción-Mundo-Dasein, tanto en su interpretación fenomenológica sobre Aristóteles, como en su análisis de la mundaneidad del Dasein en SuZ. De esta manera nos proponemos circunscribir su respectiva perspectiva y comprensión, en vistas a nuestro posterior análisis.

\section{I.1. HACIA UNA PRAXIS ORIGINARIA}

En estos trabajos, con el objeto de retomar las experiencias originarias del pensar, desarrolla sus lecturas de la filosofía práctica aristotélica, principalmente a partir de la distinción entre praxis, phrónesis y poiésis, como disposiciones o modalidades de acción (Aristóteles 1993, VI, 5, 1140 a). Estas lecturas de Heidegger confluyen en la reelaboración, re-jerarquización

4 Las puntualizaciones en este manuscrito, recuperado en los años 80 , se desarrollan en cierta continuidad con sus cursos de Friburgo Phänomenologische Interpretationen zu Aristoteles (1921-1922). En la misma línea, sus elaboraciones respectivas en Heidegger 1924 (en Marburgo) tienen sus desarrollos en Heidegger 1924-1925.

5 Específicamente, desde sus lecturas de los libros VI de Ética a Nicómaco (1923) (1140 a25-1145 a 10) y IX de Metafísica (1990) de Aristóteles desarrollados en: Heidegger 1921-1922; Heidegger 1924; Heidegger 1924-1925, entre otros.

6 Cf. Gadamer 2003, pp.76s.; Volpi 2012, pp.32ss.; Thanassas 2012, pp.43s.; Di Pego 2015, pp.32-7, entre otros. 
y torsión de tales distinciones, sobre el trasfondo de la búsqueda de ámbitos ontológicos originarios, a partir de un enfoque que destaca el carácter de uso y disponibilidad como los modos primarios en que se nos dan los entes (Heidegger 1921-1922, p. 398). ${ }^{7}$ En este marco, destaca que la filosofía como práctica del pensar, para su preguntar y despliegue respectivo, debe remitir ineludiblemente a la actividad y experiencia humana inmediata.

En efecto, Heidegger interpreta aquellas disposiciones aristotélicas en el horizonte de su indagación en torno a la unidad ontológica-estructural del Dasein. Así, frente a las cinco determinaciones del alma que Aristóteles distingue para el acceso o la apertura a la verdad (Aristóteles 1993, VI, 3, 1139 b), Heidegger delimita dos formas: el ámbito práctico (logistikón) (relacionado con la téchnê y la phrónesis) y el teórico (epistemonikón) (relacionado con la episteme y a la sophia) (Heidegger 1924, pp. 28-31).Esta distinción le permite reelaborar la jerarquización aristotélica, en su consideración del ámbito práctico contingente de la poiésis y la praxis, a partir del reconocimiento de ámbitos originarios y derivados.

Específicamente, desde las formas de conocimiento distinguidas por Aristóteles (episteme/sophia para la theoría; téchnê para la poiésis y phrónesis para la praxis), Heidegger se contrapone a la consideración eminente de la theoría por su vinculación con la contemplación de lo divino y lo eterno, cuyo carácter necesario lo distingue de los asuntos humanos (Heidegger 19211922,pp. 388-90[74-6]). ${ }^{8}$ Nuestro autor entiende que estas elaboraciones no se han constituido desde el ámbito fenomenal propio de la existencia humana, pues Aristóteles no distinguió su unidad ontológica como temporeidad originaria, lo cual condujo a un tratamiento inadecuado del mundo humano de la praxis. ${ }^{9}$

Frente a esta comprensión Heidegger contrapone su interpretación de la theoría como modalidad de praxis secundaria, respecto al trato práctico con los entes en la cotidianidad del mundo, en el marco de la estructura ontológica del Dasein, la cual permite unificar tales distinciones en una praxis originaria. ${ }^{10}$ Ello implica un cambio de acento fundamental: a diferencia del ámbito de lo eterno, la vida humana se caracteriza por un dinamismo y contingencia que requiere un modo propio de acceso (Heidegger 1921-1922, pp.376ss. [60ss.]).

En este sentido, la consideración aristotélica del par praxis-phrónesis, donde distingue el fin práctico intrínseco a la acción virtuosa (respecto de la externalidad propia de la téchnê), es reelaborada por nuestro autor, al

7 Cf. Heidegger 1924, pp.25s.

8 Cf. Aristoteles 1990, I, 980b-983a25 y Aristóteles 1993, X, 8, 1178a10-b23.

9 Cf. Taminiaux 1989, pp. 185s.

10 Cf. Volpi 1994, pp.380ss. Cf. Villarroel 2006, p.82. 
comprender la praxis desde la irreductibilidad de la facticidad en la que se halla el Dasein, con sus relaciones y proyecciones respectivas. En este contexto, mientras la deliberación/phrónesis aristotélica se articula irreductiblemente desde la interacción entre los hombres (Aristóteles 1993, VI, 1141b), para Heidegger, la resolución proyectiva del Dasein se despliega primariamente en el ámbito de las dinámicas de la mismidad. ${ }^{11}$

Desde este enfoque, la praxis, en su carácter originario, articula aquellas tres formas de acción determinadas, perdiendo con ello la determinación estática atribuida por esta tradición contemplativa: las relaciones cotidianas con los entes remiten, en definitiva, a las posibilidades de acción entendidas como modalidades de despliegue del ser-posible del Dasein. Esta torsión y re-jerarquización implica un doble desplazamiento: por un lado, el paso del significado del ser en la orientación aristotélica a la producción (a partir de la relación téchnê-poiésis) hacia la consideración primaria del uso, en el contexto instrumental del Dasein como ser-en-el-mundo (Heidegger 1921-1922, p.373 [57]); por otro, esta ontologización desarticula la praxis de su tradicional vinculación con la deliberación y la ética.

En esta perspectiva y sus desplazamientos, el mundo deja ya de comprenderse como conjunto objetivo de entes, para abrirse como un ámbito donde se despliegan las posibilidades prácticas del obrar, cuyas remisiones remiten en última instancia al poder-ser (Seinkönnen) o proyecto del Dasein. Con ello, no solo la vida se comprende irreductiblemente unida al mundo, sin pretensión de exterioridad contemplativa y pura respecto de la experiencia, sino que también se inscribe en una teoría del obrar que supone un desplazamiento de aquella comprensión tradicional restringida a la acción efectiva y presente. Estas consideraciones tienen sus específicos desarrollos en las elaboraciones de SuZ.

\section{I.2. MUNDANEIDAD Y PRAXIS EN SEIN UND ZEIT}

Podemos distinguir una articulación con esta perspectiva, especialmente en los parágrafos 14-19 de SuZ, a partir del desarrollo de la mundaneidad del Dasein, en el marco de la denominada dimensión práxica de la utilizabilidad (Ihde, 1979, pp.38s). Allí, Heidegger parte de los dos modos en que los entes se nos abren en el mundo circundante: el uso (Gebrauchs) (junto a la utilidad -Dienlichkeit-) y el trato (Umgang). Desde allí, analiza la modalidad primaria de praxis articulada en la estructura ontológica del cuidado (Sorge). Precisamente desde esta estructura originaria, Heidegger distingue la ocupación o trato cotidiano (Besorgen), como modalidad específica en que

11 Cf. Di Pego 2015, pp.37ss.Cf. Butierrez 2020.

Contrastes vol. XXV-Nº2 (2020) 
se nos dan los entes en el mundo. Con ello, pone el acento en la apertura del mundo a partir de la relación ontológica entre praxis y cuidado. ${ }^{12}$

Este carácter primario del uso se articula con la distinción de los modos de ser que Volpi vincula con sus anteriores elaboraciones en torno a la herencia aristotélica. En efecto, la ontologización de la theoría, poiésis y phrónesis se profundiza al ser equiparables con los modos de ser del carácter de simpleestar-ante (Vorhandenheit), del estar a la mano/utilizabilidad (Zuhandenheit) y el cuidado, respectivamente. ${ }^{13}$ Ello le permite insistir en sus comprensiones respecto de la theoría: cuando el Dasein se dispone teóricamente, el ente se le manifiesta como meramente presente en el mundo, esto es, al modo del simple-estar-ante.

De esta manera, la distinción fundamental entre la presencia y el estar a la mano demarca el ámbito de todo aquello que es en el mundo, al tiempo que permite especificaciones de las respectivas relaciones de tales formas de ser con el Dasein. En esta tríada, el trato con los útiles revela la mundaneidad con sus remisiones de significatividad (Heidegger SuZ, pp.61s. [87s.]), lo cual ha sido interpretado como un preludio para sus elaboraciones posteriores en torno a la técnica (Dreyfus 2006, pp.58ss).

En el marco de este entramado remisional, ${ }^{14}$ el saber inmediato se pone de manifiesto como conocimiento preteorético: la efectividad de la acción revela un saber del trato y comprensión originaria ya abierta en todo uso determinado (Heidegger SuZ, 68s. [96s.]).En este sentido, solo la utilizabilidad del ente permite comprobar el saber de uso, lo cual puede ser entendido como un pragmatismo del mundo de la vida que remite, ya no al conocimiento de verdades necesarias, sino a un saber práctico ligado a la acción cotidiana. ${ }^{15}$

En línea con sus elaboraciones de 1922/1925, el trato operativo y técnico en el mundo circundante da cuenta de una finalidad del obrar cuyo empuje no se orienta ya hacia una causa final, sino al tener que ser del Dasein, en una proyección al modo de una pregunta abierta cuya apelación a ser, posibilita su respuesta (Rubio 2003, p.318). Ello permite poner de manifiesto el modo específico de ser del Dasein en su estructuración unitaria y originaria, no reductible a la mera presencia.

En suma, con estos abordajes y elaboraciones, nuestro autor se propone dejar de lado la distinción tradicional entre teoría y praxis, para distinguir esta última como vía de acceso eminente para las relaciones del Dasein en

12 Cf. Heidegger SuZ, pp.67-72[94-9]. Cf. Heidegger 1923; pp.101-4[128-32]; Heidegger 1924, pp.352s.

13 Cf. Volpi 2007, pp.36ss. Según este intérprete, también es posible distinguir analogías estructurales entre la praxis y el Dasein (Volpi 2012, pp.75-152).

14 Cf. Heidegger SuZ, p.69 [96s.].

15 Cf. Gethmann 1998, pp.142-6. Cf. Chiappe 2012,568s. 
el mundo, en parte dando cierta continuidad a la filosofía práctica tradicional y en parte planteando una torsión fundamental del marco comprensivo de la praxis. En este marco, Heidegger subraya que las relaciones fácticas en el mundo distinguen el ámbito de análisis de la filosofía, respecto de aquél propio de las ciencias. En este sentido, la labor filosófica debe desplegar su preguntar a partir de la experiencia fáctica de la vida, atendiendo su propia modalidad temporal. Precisamente encontramos una singular articulación de estas cuestiones en su seminario de finales de los años 20 .

\section{El DESPERTAR DEL ABURRIMIENTO PROFUNDO EN 1929-1930}

En el siguiente apartado analizaremos las relaciones entre la figura del despertar y el discurrir del preguntar filosófico que Heidegger desarrolla en la primera parte de su seminario de 1929-1930. ${ }^{16}$ A nuestro entender, allí se articulan elementos fundamentales de su comprensión en torno a la acción y la praxis. En tal sentido, la modalidad en que despliega el preguntar filosófico respecto de cuestiones eminentes de la metafísica articulan el pensamiento de Heidegger en una modalidad práctica que nos permite ampliar la comprensión de su alcance y medida.

Aquí nos detendremos, en primer lugar, en sus especificaciones prácticas respecto a la necesidad de despertar un temple de ánimo fundamental para el despliegue de preguntas metafísicas fundamentales. Luego, mediante un contraste con las modalidades de acción con las que Heidegger se diferencia, buscaremos circunscribir el alcance de su procedimiento, los resultados obtenidos y la caracterización del pensamiento resultante, en el marco de la constelación temática que orienta nuestros análisis.

\section{II.1. EL ESTATUTO PRÁCTICO DEL DESPERTAR}

La experiencia del preguntar descripta en los primeros parágrafos de Die Grundbegriffe der Metaphysik (GbM), remite al carácter irreductible de la relación con el mundo, siendo el propio despliegue de este preguntar esencial la modalidad eminente de acción que subraya Heidegger. Ello requiere como condición que aquél que pregunta experimente un arrebatamiento por parte de aquello que se pregunta, como indicio de un preguntar auténticamente enraizado en la experiencia. En este marco, Heidegger subraya que el filosofar tiene como objeto despertar (Weckung) tal arrebatamiento primario, señalando así el carácter originario del temple de ánimo respecto del filosofar.

Este recurso de la metáfora del despertar también fue utilizado en Was

16 Aquí abordaremos su conferencia de julio Was ist Metaphysik? (1929) y su seminario posterior Die Grundbegriffe der Metaphysik. Welt - Endlichkeit - Einsamkeit (1929-30). 
ist Metaphysik?, aunque con un cambio de acento fundamental: allí refiere a un ocasionamiento nimio e impersonal que despierte el profundo alcance de la angustia originaria, reprimida y adormecida en el Dasein (Heidegger 1919-1961, p.118 [104s.]). En GbM, si bien se propone tomar distancia de la perspectiva tradicional de la conciencia o el yo, su articulación de la figura del despertar no parece exenta del sesgo voluntarista del que busca distanciarse. Por ello, la primera parte de esta indagación de Heidegger irá de la mano de la especificación de la labor filosófica junto a una aclaración del sentido de este despertar, con el objeto de despojarlo de aquella significación voluntarista tradicional (Ibid., pp.89-116[89-110]).

En detalle: nuestro autor distingue que el hacer humano también se mueve en situaciones que no domina, sin certezas definitivas, donde se pone en juego aquello esencial de su propia existencia. En el marco de estas experiencias profundas, la voluntad debe contenerse en pos de una apertura en el marco radical de estas experiencias. Precisamente aquí inscribe el carácter experiencial del preguntar filosófico, en su apertura acontecimental y originaria, cuya modalidad vinculante y peligrosa lo distingue del proceder científico.

En esta caracterización de la experiencia, retoma sus análisis anteriores sobre los términos griegos physis, legein y logos, para poner de manifiesto una comprensión originaria que no encubre el ocultamiento y retracción correlativas a todo ente que viene a la presencia. ${ }^{17}$ En este ámbito originario para la indagación filosófica, subraya la posición de aperturidad y su temple, sin descuidar sus condiciones esenciales:

[...] [A]ntes que nada tenemos que hacer surgir estas preguntas en su necesidad y posibilidad desde un temple anímico fundamental y tratar de conservarlas en su autonomía e inequivocidad. Por consiguiente, este preguntar lo realizamos cuando nos ponemos a despertar un temple de ánimo fundamental de nuestro filosofar. Esta es la primera y auténtica tarea fundamental de nuestra asignatura y el comienzo de un auténtico filosofar viviente (Ibid.,p.87 [86]-el subrayado es nuestro-).

Pero ¿en qué sentido se requiere de un despertar? ¿Supone ello una fuerza voluntaria que manipula, bajo cálculo, su propio templarse? En este punto de ambigüedad podemos encontrar diversas interpretaciones: por un lado, aquellas que leen esta figura como la exigencia de un estar despierto para sí mismo, en vistas de un acceso privilegiado a la verdad (Catoggio 2008, pp.15; 21); en la misma línea, distinguimos lecturas que resaltan la carácter operatorio del despertar un temple fundamental que capacite para poder

17 Cf. Ibid., p. 41[53]. Cf. Heidegger SuZ, pp.28-39 [51-62]. 
comenzar en una nueva época histórica (Held 2015, p.p.27); por otro lado, destacamos interpretaciones que lo entienden como una invitación del propio Heidegger orientada al auditorio o los lectores (Padilla-Teruel 2018, p.299). ${ }^{18}$ A nuestro juicio, estas lecturas descuidan que la significación y la modalidad de acción allí articulada se encuadran en un sentido más bien originario.

En efecto, para Heidegger el temple de ánimo no puede forzarse arbitrariamente sino tan solo dejar que despierte lo que duerme, equiparable con dejarlo ser en tanto temple de ánimo (Heidegger 1929-1930, p.92s. [92]). El accionar calculador y voluntario, al que nuestro autor se opone, se articula en este adormecimiento para así detener un temple fundamental: un modelo del obrar de época que se propone interferir con todo aquello que ponga en juego la propia existencia.

En este sentido, la figura del despertar se despega de su anclaje tradicional en el par consciente/inconsciente, dado que las remisiones a la conciencia no hacen más que alterar e intervenir aquello a lo que se busca acceder. Incluso éstas se revelan en su carácter derivado, pues el temple de ánimo modaliza de un modo primario la relación con los entes, con el sí mismo y con los otros. Es el cómo en el que estos se articulan y en el que nos encontramos en la existencia. Por ello, no puede articularse en un proceder racional-consciente o de cálculo de acciones, sino en un despertar como modalidad propia de acción.

Estas distinciones permiten a Heidegger subrayar que despertar un temple fundamental requiere conocer la situación actual y la época histórica. Como sostiene un intérprete, aquí puede reconocerse un cambio en la perspectiva de análisis: el paso de una indagación centrada en la relación con el sí mismo hasta Sein und Zeit, a la consideración del temple de una época, lo cual se orienta hacia la crítica de la cultura y de la técnica, que desarrolla ampliamente en sus trabajos posteriores de los años 50 (Catoggio 2008, p.22).

En especial, nuestro autor se contrapone a los estudios culturales de época que versan sobre la situación de la existencia en Occidente. A partir de una breve indagación de las interpretaciones de Spengler, Klages, Scheler y Ziegler, ${ }^{19}$ reconoce como base común la problematización de la relación Vida-Espíritu, enmarcada en la comprensión nietzscheana de lo dionisíaco y lo apolíneo. Nuestro autor rechaza estos diagnósticos y pronósticos, pues entiende que no alcanzan a interpelar al Dasein, ni a poner en juego en su propia situación: como perspectivas no vinculantes, no se hallan arrebatadas primariamente por aquello en lo que indagan. Frente a esto, Heidegger

18 Cf. Lythgoe 2014b, p.208.

19 Cf. los análisis de las perspectivas de estos autores en Rodríguez 1991, pp.196ss. 
se pregunta por aquello que sucede con nosotros en la época, como una modalidad propia y genuina de exponer la propia situación.

En el contexto de esta pregunta, destaca que el aburrimiento expresa el modo epocal en que somos afectados, entendido como una constelación afectiva que impregna toda una época histórica (Adrián 2014, p.37). Este temple de ánimo no solo abre de manera inmediata el conjunto de la situación de la existencia, sino también impide los intentos racionales de determinarla. En tal sentido, destaca que la programática de acción de aquellas perspectivas culturales y su respectiva asignación de roles históricos para el accionar humano, en última instancia se basan en una indiferencia y aburrimiento respecto de la propia existencia, templando del mismo modo las distintas relaciones en el mundo. ${ }^{20}$ Frente a ello, sus análisis del aburrimiento ponen de manifiesto la singular modalidad activa implicada en el despertar.

\section{II.2. Mundo y DASEIN EN EL ANÁLISIS DEL ABURRIMIENTO}

Nuestro autor subraya que el aburrimiento profundo (tiefe Langeweile) es un temple de ánimo fundamental (Grundstimmung) ${ }^{21}$ cuya modalidad vincular cotidiana se da en el modo de la huida, esto es, como intervención práctica para intentar detenerlo, eliminarlo o hacer que se duerma y con ello, mantenerlo a su alcance bajo cálculo. Nuestro autor propone un movimiento opuesto a esta tendencia de adormecimiento: un despertar el temple de ánimo, que no significa hacer primero que se despierte, sino hacer que esté despierto, preservarlo del dormirse (Einschlafen) (Heidegger 1929-1930, p.118 [112]). En esta circularidad activa nuestro autor enfatiza una dinámica no voluntaria: el temple condiciona la experiencia, al tiempo que lo dado en ella modela el temple.

Aquí se pone de manifiesto una experiencia pura del tiempo. ${ }^{22} \mathrm{La}$ dimensión temporal implicada en dicho temple se torna evidente en la expresión alemana que literalmente significa: el momento (Weile) que se hace largo (lang), lo cual ya prefigura una diferencia fundamental con el instante (Augenblick), cuyo término sugiere una temporalidad atravesada por las dinámicas temporales de la finitud humana, tal y como también se especifica

20 Lo cual articula en un preguntar filosófico: ¿sucede al cabo con nosotros que un aburrimiento profundo se mueve de un lado a otro en los abismos de la existencia como una niebla silente? (Heidegger 1929-1930, p.115 [109]). Cf. Heidegger 1919-1961, p.110 [98].

21 Held entiende relevante esta denominación en remplazo de la disposición afectiva fundamental (Grundbefindlichkeitde), utilizada en trabajos anteriores de Heidegger: a diferencia de la consideración de la angustia, ella remite a una disposición para una experiencia de la constitución esencial de nuestra época, en Held 2015, p.22.

22 Cf. Kawahara 1987, 91ss.Cf. Van Dijk 1991, pp.100ss. 
en este seminario de Heidegger.

En efecto, el procedimiento de nuestro autor se encuadra en una relación con el temple de ánimo, cuyo hilo conductor es indagar en el ámbito donde nos oponemos a él: el pasatiempo (Zeitvertreib). De este modo, busca mantenerse en la inmediatez de la experiencia cotidiana, sin imponer acciones determinadas: primero encuentra necesario determinar el temple y luego, despertarlo.

Por esta vía, distingue tres formas de aburrimiento, desde expresiones/ experiencias correlativas: el aburrirse con algo (das Gelangweiltwerden von etwas); el aburrirse en/por algo (das Sichlangweilen bei etwas) y el uno se aburre (o me aburro) (es ist einem langweilig), en referencia a un aburrirse sin más (das Sichlangweiligen). Tales distinciones permiten dar cuenta de los diversos grados de profundidad en la que se haya implicada la existencia. En el primer caso, parte del ejemplo cotidiano de llegar antes de horario a la estación de tren; en el segundo, de la asistencia a una velada para distraerse del trabajo y en el tercero, destacando la dificultad que implica su determinación, esboza el caso de caminar por una gran ciudad, un domingo por la tarde. Desde cada uno de ellos indaga en aquél pasatiempo que puede usarse para oponerse al aburrimiento. En este marco, los dos momentos estructurales de estas formas de aburrimiento son el dar largas (Hingehaltenheit) del tiempo (como oprimir de éste) y el ser dejados vacíos (Leergelassenheit) por las cosas (o por el ente en su conjunto). Por medio de ambas, Heidegger pone en evidencia las relaciones del temple fundamental con el mundo y el sí mismo, en consideración del carácter proyectivo del Dasein.

En tal sentido, nuestro autor pone énfasis en que el aburrimiento profundo implica un bloqueo y vaciamiento del poder-ser en el instante, correlativo con una indiferencia del mundo y los entes, articulado con la indeterminación de aquello que ocasiona el aburrimiento. ${ }^{23}$

Aun así, esta indeterminación y bloqueo no deja otra opción más que escuchar al temple (Basso 2014, p.287), en el marco de una espera cuyo carácter arrebatador (y, por ello, propiamente auténtico) nos depone del modo de ser en la cotidianidad. Este carácter coercitivo y de sustracción del Dasein en su cotidianidad se encuentra prefigurado en sus elaboraciones anteriores en torno a la angustia, con la cual emerge la falta de significatividad del mundo. Pero la especificidad del aburrimiento reside en su modo de proporcionar herramientas para saber moverse en la profundidad de la existencia (Heidegger 1929-1939,p.198 [171]). Con ello, nuestro autor subraya un saber-hacer que orienta este procedimiento del despertar.

Sin embargo, con este temple la mismidad del Dasein se encuentra radicalmente modificada, pues éste desactiva su poder-ser fáctico: la 
indeterminación bloquea las posibilidades del obrar, lo cual co-implica un reconocimiento de las mismas en su potencialidad. Precisamente por ello, nuestro autor insiste en el dejar-ser del despertar: permitir que el temple vibre hasta el final pone en evidencia lo posibilitador en general y el instante, el ámbito originario de temporeidad (Zeitlichkeit) del Dasein que el aburrimiento anula, al mismo tiempo que anuncia. ${ }^{24}$ De este modo se revela la temporeidad como aquello que impide la manifestación del ente y su accesibilidad.

Desde estas consideraciones, Heidegger parece ampliar los resultados de sus trabajos de comienzo de los años 20 respecto a la problemática de la temporeidad, que confluye en SuZ con su distinción como ámbito originario que unifica las estructuras fundamentales del Dasein (Pocai 1996, pp.193s.).No obstante, la consideración del carácter horizontal de la temporeidad en este seminario (no ya desde los éxtasis temporales del Dasein), ${ }^{25}$ así como también el alcance radical de la manifestación del mundo y del sí-mismo en el aburrimiento profundo, son indicios de un marco transicional en la perspectiva de Heidegger, respecto de su enfoque hasta SuZ.

En este sentido, destacamos la lectura de Agamben para quien el aburrimiento profundo se presenta aquí como un operador metafísico que permite el acceso al mundo humano, en el marco de un proceso de antropogénesis que se haya vinculado con el despertar heideggeriano, tal y como subraya:

El Dasein es simplemente un animal que ha aprendido a aburrirse, se ha despertado del propio aturdimiento y al propio aturdimiento. Este despertarse del viviente a su propio ser aturdido, este abrirse, angustioso y decidido, a un no abierto, es lo humano (Agamben 2016, p.129-el subrayado es del autor-)

Siguiendo esta lectura del despertar como apertura podemos destacar que la modalidad del obrar aquí articulada remite a una figura irreductiblemente inscripta en la experiencia del Dasein en el mundo, cuyo movimiento propio se articula en el preguntar filosófico.

\section{II.3. HACIA UNA AUTÉNTICA PRÁCTICA FILOSÓFICA}

En línea con el oficio del poeta, esta figura del preguntar de Heidegger y su tentativa de despertar derivan en un dejarse templar para escuchar lo esencial de dicho temple. En este sentido, despertar implica no-actuar-encontra, como significación activa desde un ámbito más originario que el obrar pasivo y activo (Heidegger 1929-1930,p.240 [206]).

24 Heidegger distingue entre la temporeidad (Zeitlichkeit) o determinación del ser del Dasein por el tiempo y la temporalidad (Temporalität) o determinación del ser mismo por el tiempo.

25 Cf. Lythgoe 2014 a, pp.770ss. 
Esto señala el carácter primario e irrebasable del componente afectivo de la labor filosófica, entendida como un modo originario de asidero y de experiencia, donde aquél que pregunta es desplazado de su posición inicial y redirigido por el mismo preguntar, que simultáneamente desencadena su fuerza interna. Así, la potencia del preguntar se contrapone al programatismo del entendimiento común de aquellas perspectivas culturales de época, que no solo apuntan con su praxis a las respuestas en su carácter fijo y definitivo, sino que se proponen esquivar la opresión esencial (wesenhaften Bedrängnis) de la existencia y el vacío en su conjunto, como los marcos propios del aburrimiento profundo.

Este cambio disposicional requiere para Heidegger de una apertura anterior, un permitir-ser-dejados vacíos para el instante y el misterio (Geheimnis) lo cual, en definitiva, permite caracterizar el cómo (wie) de la situación de época, tal como específica en este fragmento:

[...] [E]n nuestra existencia falta el misterio, y con ello falta el horror interno que todo misterio lleva consigo y que le da a la existencia su magnitud. Lo que oprime en el fondo y lo que nos deja hondamente vacíos es la falta de opresión, es decir, el vacío que aburre en el fondo. Esta falta de opresión se oculta en apariencia, más bien se testimonia justamente en las ocupaciones del ajetreo actual. Pues, en último término, en todo el organizar y hacer programas y probar hay un general goce saciado en una falta de peligro (Ibid., p.244s. [209]).

Aun así, Heidegger destaca que el filosofar solo conduce al borde de la posibilidad: el salto concreto se articula en el instante, anunciado por el aburrimiento profundo mediante su anulación. En tal sentido, el preguntar esencial implica un ponerse en camino para ser despertado.

Estas especificaciones en torno a la práctica filosófica anticipan sus análisis posteriores del mundo de la técnica, donde el tiempo se muestra ya domesticado por la racionalidad tecnológica, tal como lo señala Adrián Escudero:

Las reflexiones en torno al aburrimiento ponen de manifiesto la verdadera naturaleza de la racionalidad tecnológica: su voluntad de dominio y control. El mundo y las relaciones humanas quedan reducidos a puras mercancías. En este sentido, el aburrimiento ofrece la posibilidad de romper con los engranajes de un mundo públicamente vertebrado. Cuando caemos presos del aburrimiento, la racionalidad tecnológica tiene que hacer frente a algo que no puede controlar. Y esto significa que de alguna manera nos liberamos de su dominio (Adrián 2007, p.367). ${ }^{26}$

26 Cf. Adrián 2014, p.40. 
En suma, el aburrimiento profundo permite a Heidegger destacar la propia existencia del hombre como un peso que debe asumir, al modo de una carga ineludible. Desde allí, dejarse templar por el aburrimiento profundo implica una apelación coextensiva con la resolución y proyección del Dasein, abriendo un ámbito para el encuentro con el misterio y el peligro de la existencia. En este sentido, el despertar como dejar-ser se liga al filosofar como preguntar esencial: una modalidad originaria de acción que abre las condiciones para un actuar auténtico en la existencia humana.

\section{A MODO DE CONCLUSIÓN}

Como colofón, puntualizaremos brevemente los aspectos fundamentales de nuestros análisis, con el objeto de circunscribir la perspectiva del obrar y la acción que Heidegger articula en esta primera parte de su seminario de 19291930. Desde allí buscaremos distinguir en qué medida ella puede entenderse como una elaboración de tipo fronteriza, entre sus elaboraciones en torno a SuZ y su perspectiva después de la Kehre.

En primer lugar, establecimos un recorrido puntual por su recepción de los textos aristotélicos, en el marco de sus clásicas reelaboraciones entre 1921 y 1927. Allí destacamos la elaboración originaria de la praxis y el consecuente carácter derivado de la theoría. Desde esta comprensión, Heidegger se propone aclarar el punto de partida adecuado para la indagación filosófica: la experiencia inmediata en la facticidad del mundo. En este marco, el proyecto de una ontología fundamental, cuyo programa se consolida en SuZ, confluye en la distinción de la temporeidad como el ámbito unitario y primario de la estructura ontológica del Dasein, en el camino de la pregunta por el ser. Una vez aclarado el entramado estructural respectivo, Heidegger se ve forzado a retomar su camino del pensar, al modo de una transición respecto a la perspectiva de la metafísica tradicional y su herencia conceptual. Desde 1930, ello se articula con un enfoque puesto en las dinámicas relacionales Dasein-Ser en el horizonte del Ereignis. En dicho recorrido, nuestro autor busca desligarse de todo residuo subjetivista para pensar dichas relaciones, especialmente en lo que respecta a la constelación Acción-Mundo-Dasein.

Uno de los modos específicos en que Heidegger indaga y piensa aquellos textos tradicionales, remite a una re-jerarquización y reelaboración comprensiva que prefigura la deconstrucción derrideana, que nuestro autor articula a partir de la destrucción y desmontaje relacional de los conceptos considerados. Desde aquí, su consideración originaria de la praxis permite establecer articulaciones con la práctica filosófica. En efecto, la modalidad de orientación ontológica, tanto en dichas reelaboraciones como en la analítica de la mundaneidad en SuZ, se manifiesta como indicio de un estilo práctico de 
indagación filosófica correlativo a la articulación de su propio marco teórico de la praxis, donde las relaciones irreductibles y prácticas en el mundo no quedan reducidas a lo meramente presente y verificable en acto.

En segundo lugar, nos centramos en un análisis de la primera parte de su seminario de 1929-1930, a partir de la consideración de la figura del despertar en su análisis del aburrimiento profundo, el cual permite poner de manifiesto el mundo, los entes y la mismidad proyectiva del Dasein en su conjunto. La orientación de nuestras lecturas fue dada por la indagación en torno al estatuto práctico de dicha figura. Por esa vía, vimos que el despertar remite a un ámbito de anterioridad, preparatorio para un obrar auténtico, el cual se contrapone a la modalidad voluntaria e incluso compulsiva del actuar cotidiano. Heidegger da cuenta de un despertar como dejar-ser aquello que es forzado a dormirse, lo cual implica un no-actuar-en-contra y dejarse templar por el aburrimiento. De esta manera, podemos distinguir en esta figura metafórica elementos en desplazamiento respecto de la centralidad puesta en el Dasein en sus elaboraciones anteriores, profundizando en el énfasis puesto en las dinámicas anteriores y primarias.

A nuestro entender, esto implica una continuidad con la orientación ontológica de su indagación filosófica, al tiempo que desplaza su enfoque a dinámicas correlativas que no tienen a la conciencia ,el ego o el sujeto como punto de partida, aspectos que anticipan sus elaboraciones posteriores en torno al mundo de la técnica.

Aquí insiste en las bases fácticas y situacionales para el preguntar filosófico, al tiempo que subraya el componente irreductiblemente templado desde el que se abre la proyección de su labor, en el marco de una dinámica experiencial donde la constelación Acción-Mundo-Dasein se ve relacionalmente desplazado. En tal sentido, para Heidegger el filosofar representa una modalidad eminente de pensar la situación histórica, frente a las perspectivas y diagnósticos culturales.

En el marco de estos aspectos considerados, podemos sostener que las respectivas implicancias del actuar y el pensar se despliegan de un modo transicional y fronterizo entre dos perspectivas o enfoques de sus elaboraciones, en el marco del estatuto práctico del pensar heideggeriano caracterizado por dinamizar los resultados de su propio recorrido.

En sus trabajos posteriores, el estatuto de la praxis y de la acción se enmarca de manera creciente en la dilucidación de estos ámbitos pre-subjetivos y preteóricos, como condiciones irreductibles de todo obrar determinado. En este sentido, la apertura primaria de los temples de ánimo fundamentales en y desde la experiencia en el mundo, le permiten subrayar posiciones anímicamente abiertas como condiciones de posibilidad de un obrar auténtico que responda a la exhortación del otro inicio del pensar occidental. 
De esta manera, destacamos el carácter relevante de estas elaboraciones de 1929-1930 para seguir precisando el estatuto de la praxis en la obra de Heidegger. En esta línea, los análisis comparativos entre la piedra, el animal y el Dasein, tal y como lo desarrolla en la segunda parte del seminario, también pueden aportar elementos para vislumbrar el alcance de las elaboraciones heideggerianas del pensamiento y la praxis en sus trabajos de madurez.

\section{REFERENCIAS BIBLIOGRÁFICAS}

ADRIÁN, J. 2007: Hacia una fenomenología de los afectos: Martín Heidegger y Max Scheler, Thémata. Revista de Filosofía, 39, pp.365-368.

ADRIÁN, J. 2010: Heidegger y la genealogía de la pregunta por el ser, Barcelona, Herder.

ADRIÁN, J. 2014: El aburrimiento, o de la importancia de los estados de ánimo en la filosofia de Heidegger, Graffylia 18, pp. 33-42.

AGAMBEN, G. 2016: Lo abierto, el hombre y el animal, Bs As. Adriana Hidalgo ed. ARISTÓTELES. 1990: Metafisica, trad. Valentín García Yebra, Madrid, Ed. Gredos. ARISTÓTELES, 1993: Ética Nicomáquea. Ética Eudemia, Trad. Julio Pallí Bonet. Madrid: Gredos.

BASSO, L. 2014: Aportes para una interpretación de la estructura del Dasein de acuerdo a su carácter bidimensional, Areté. Rev. de Filosofía, V.26, 2, pp.273-293.

BUTIERREZ, L. 2020: Caminos hacia la alteridad. La comprensión del otro en las elaboraciones de Heidegger en torno a Sein und Zeit, Anales del seminario de historia de la filosofía, en prensa.

CALLE ZAPATA, M. 2016: Martin Heidegger y el intento por pensar la esencia de la técnica como una reorientación en el Ethos, Revista Perseitas, 4,1, pp. 41-61.

CATOGgiO, L. D. Parente 2008: Angustia y aburrimiento. Reflexiones sobre un desplazamiento temático en el primer Heidegger, Ergo. Revista de Filosofía, 22-23, pp.7-24.

CHIAPPE, A. 2012: La filosofía de la técnica implícita en el primer Heidegger, Rev. Filos., Aurora, Curitiba, v. 24, n. 35, pp. 549-579.

DE LARA, F. 2014: El gesto político de las filosofias de Heidegger. Contribución a un debate actual, Veritas, $\mathrm{N}^{\circ} 31$, pp.73-86.

DI PEGO, A. 2015: Martin Heidegger y la rehabilitación de la filosofia práctica aristotélica: distanciamientos y continuidades, Aporía Revista Internacional de Investigaciones Filosóficas; Lugar: Santiago de Chile; Año, pp. 29 - 47.

DI PEGO, A. 2019: En torno al pensamiento: la disputa de Hannah Arendt con Martin Heidegger, Tópicos, Revista de Filosofia 56, enero-junio, pp. 197235.

DREYFUS, H.L. 2006: Entre la 'tekné'y la técnica: el ambiguo lugar del útil en Ser y Tiempo, en La técnica en Heidegger vol. $2^{\circ}$, Santiago de Chile, Universidad Diego Portales. 
GADAMER, H. G. 2003: Heideggers 'theologische'Jugendschrift, en: Heidegger, Gadamer; Gunther. (Hrsg.) Phänomenologische Interpretationen zu Aristoteles. Ausarbeitung für die Marburger und die Göttinger Fakultät (1922) . Stuttgart: Reclam.

GETHMANN, C. F. 1998: Heideggers Konzeption des Handelns in 'Sein und Zeit', en GethmannSiefert, A. /Pöggeler, O. Heidegger und die praktische Philosophie, Suhrkamp, Frankfurt.

HEIDEGGER, M. 1919-61: Wegmarken [T.C.: Hitos, Madrid, Alianza, (2007), trad.: Helena Cortés y Arturo Leyte]. (GA 9).

HEIDEGGER, M. 1921-1922: Phänomenologische Interpretationen zu Aristoteles zu Ontologie (Anzeige der hermeneutischen Situation) ed. G. Neumann, 2005. [T. parcial: Interpretaciones fenomenológicas sobre Aristóteles. Indicación de la situación hermenéutica (Informe Natorp), Madrid, Trotta, (2002), Trad: Jesús Adrian Escudero] (GA62).

HEIDEGGER, M. 1923:Ontologie. Hermeneutikder Factizität [T.C.:2008:Ontología. Hermenéutica de la facticidad, Madrid, Alianza] (GA 63).

HEIDEGGER, M. 1924: Grundbegriffe der aristotelischen Philosophie; Hrsg. von Mark Michalski, Klostermann, Frankfurt a/M., 2002 (GA 18).

HEIDEGGER, M. 1924-1925: Platon: Sophistes (Wintersemester), Hrsg. von Ingeborg Schüßler, Klostermann, Frankfurt a/M.(GA 19).

HEIDEGGER, M. 1927: Sein und Zeit (SuZ), [T.C.: Ser y Tiempo, Santiago, Ed. Universitaria de Chile, (1997), Trad. J.E. Rivera; Ser y tiempo, Buenos Aires, FCE, (2000), trad. J. Gaos] (GA 2).

HEIDEGGER, M. 1929-1930: Die Grundbegriffe der Metaphysik. Welt - Endlichkeit - Einsamkeit, ed. F.-W. von Herrmann, 2004 [T.C.: Los conceptos fundamentales de la metafisica. Mundo, finitud, soledad, t, Alianza Editorial, Madrid, (2007), Trad. J. A. Ciria Cosculluela] (GA 29/30).

HELD, K. 2015: Temple anímico fundamental y crítica a la cultura contemporánea en Heidegger, Co-herencia, V. 12, N. 23, pp. 13-40.

IHDE, D. 1979: Technics and Praxis, Boston studies in the philosophy of science, v. 24. Dordrecht, Boston: D. Reidel.

KAWAHARA, E. 1987: Heideggers Auslegung der Langweile, En Wisser, R. (ed. Martin Heidegger. Unterwegs im Denken. Friburgo y Munich: Karl Alber, pp. 87-110.

LYTHGOE, E. 2014 a: Disposición afectiva y temporalidad en Martin Heidegger entre 1927 y 1930, Rev. Filos. Aurora, V. 26, N. 39, pp. 759-775.

LYTHGOE, E. 2014 b: Temples de ánimo y fundamento antepredicativo de la verdad enunciativa en Martin Heidegger (1929-1930), Filosofia Unisinos, V.15 (3), pp.198-209.

PADILLA-TERUEL, R. 2018: Kairós doloroso y aburrimiento. Esperar por el preciso momento, Universitas Philosophica, V. 35(71), pp. 295-321.

POCAI, R. 1996: Heideggers Theorie der Befindlichkeit, Freiburg; München: Verlag Karl Alber.

RODRÍGUEZ, R. 1991: La ontología y las voces de la época, en Duque, Félix (ed.), 
Heidegger: la voz de los tiempos sombríos, Barcelona: Serbal.

RUBIO, R. 2003: La relevancia de la praxis en la ontología del Dasein, Areté, Revista de Filosofia Vol. XV, N0 2, pp. 303-323.

TAMINIAUX, J. 1989 : Lectures d'Ontologie Fundamentale, Grenoble: Millon.

THANASSAS, P. 2012: Phronesis vs. Sophia: On Heidegger's ambivalent aristotelianism, The Review of Metaphysics 66, pp. 31-59.

THURNHER, R. 1991: Heideggers Denken als Fundamentalethik? en Heidegger. Technik, Ethik, Politik, Reinhardt Margreiter/Karl Leidlmair (Hrsg), Würzburg, Königshausen und Neumann Verlag.

VAN DIJK, R. 1991: Grundbegriffe der Metaphysik. Zur formalanzeigenden Struktur der philosophischen Begriffen bei Heidegger, Heidegger Studien, 7, pp. 89-110.

VILLARROEL, R. 2006: Heidegger y la filosofía práctica ser y tiempo como un palimpsesto, Revista de Filosofía Volumen 62, pp. 81-99.

VOLPI, F. 1994: La existencia como praxis. Las raices aristotélicas de la terminología de Ser y Tiempo, en Gianni Vattimo (compilador), Hermenéutica y racionalidad, Barcelona, Buenos Aires, etc.; Grupo Editorial Norma, pp. 327-383.

VOLPI, F. 1996: ¿Es aún posible una ética? Heidegger y la filosofía práctica, Seminarios de filosofía N. 9, pp. 45-73.

VOLPI, F. 2007: In Whose Name? Heidegger and 'Practical Philosophy", European Journal of Political Theory 6.1, pp. 31-51.

VOLPI, F. 2012: Heidegger y Aristoteles, Bs. As., FCE.

Luis Butierrez es profesor y Doctor en Filosofia por la Universidad de La Plata y Maestrando en Clínica psicoanalítica por la Univerisad Nacional General San Martín.

Líneas de investigación:

Heidegger, Derrida, Lacan, Subjetividad, Discurso e identidad

Publicaciones recientes:

(2020). «Hacia el horizonte comprensivo de la corporeidad: Una transición entre los enfoque sde Husserl y Heidegger», Ágora. Papeles de filosofía 39(2), pp. 79-106.

(2020). Heidegger y la identidad personal. Argentina: Editorial Prometeo.

Correo electrónico: luisbutierrez@yahoo.com.ar 
